\title{
CONTACT DERMATITIS SECONDARY TO PHENYLEPHRINE EYE DROP: A CASE REPORT
}

\section{S M. Biradar 1*, Kartik Nuchhi', Akshay Kulkarni', Shivanand I. Lubadude', Kirankumar Awati', Sharan

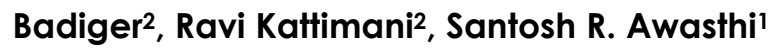

\footnotetext{
'Department of Clinical Pharmacy Practice, SSM College of Pharmacy and Research Centre, Vijaypur-586103.

2Department of Medicine, Shri B M. Patil Medical College Hospital and Research Centre, Vijaypur-586103.
}

Email: smbiradar@rediffmail.com

\begin{abstract}
Phenylephrine, a sympathomimetic drug, is commonly used in eye examinations to dilate the pupil of the eye and to differentiate scleritis from episcleritis. It is extensively used as a mydriatic agent by ophthalmologists and may cause allergic contact reactions even though it is rare. Here is a case of 46 years old female patient with acute-onset edema and erythema of both eyes associated with watering, a burning sensation, and moderate discomfort characterized by contact dermatitis. The patient develops adverse drug reaction to an eye drop containing Tropicamide $0.8 \% \mathrm{w} / \mathrm{V}+$ Phenylephrine $5 \% \mathrm{w} / \mathrm{v}$ used prior to eye fundus examination. Although uncommon, cases of allergic reaction to Phenylephrine cannot be ruled out, hence it is advisable to all the clinicians/Ophthalmologist to encourage and conduct the sensitivity test for Phenylephrine allergy, in order to minimize the possible adverse effects and ruinous consequences.
\end{abstract}

Keywords: Phenylephrine; Allergic reaction; Contact Dermatitis. 


\section{INTRODUCTION}

Phenylephrine is a sympathomimetic drug commonly used to dilate the pupil of eye and to differentiate scleritis from episcleritis in fundus examination. Tropicamide is a nonselective muscarinic antagonist commonly used for mydriasis due to its fast onset and short duration. ${ }^{1}$ Allergic contact reactions with phenylephrine are rare despite its extensive use as a mydriatic agent by ophthalmologists. ${ }^{2}$ Its action is due to a direct sympathomimetic effect upon the myoneural junction, resulting in mydriasis. ${ }^{3}$ Common adverse effects of phenylephrine include subjective burning, stinging with lacrimation, rebound hyperemia, and liberation of iris pigment into the anterior chamber. Less common, systemic adverse effects include tachycardia and elevation of systemic blood pressure..$^{5}$ Even though instances of allergic reactions are rare, phenylephrine has been reported to cause contact dermatitis as in case.

\section{Case:}

A 46-year-old Female patient was admitted to a female medicine ward with chief complaints of headache, vomiting (5-6 episodes) and syncopial attacks (loss of consciousness 2 episodes) and a known case of hypertension since two months, not on medications of hypertension. Upon patient history, she has been referred to ophthalmology department for fundoscopy and opine tests to examine hypertensive retinopathy. Administration of tropicamide $0.8 \% \mathrm{w} / \mathrm{v}+$ phenylephrine $5 \% \mathrm{w} / \mathrm{v}$ eye drops while conducting fundoscopy, After 2-3 hours of instillation the patient complained of severe burning In eyes, which further developed adverse reaction of swelling of eyes and redness surrounding both eyes (Fig. 01). Upon Rereference to ophthalmology department suspected an allergic reaction towards tropicamide $0.8 \% \mathrm{w} / \mathrm{v}+$ phenylephrine $5 \% \mathrm{w} / \mathrm{v}$ eye drops and advice milflox plus (ketorolac $0.4 \% \mathrm{w} / \mathrm{v}+$ moxifloxacin $0.5 \%$ $w / v)$ eye drops (QD) to overcome the adverse reaction. Reference of dermatology department reported the case as contact dermatitis secondary to tropicamide $0.8 \% \mathrm{w} / \mathrm{V}+$ phenylephrine $5 \% \mathrm{w} / \mathrm{v}$ eye drops.

\section{Discussion:}

Phenylephrine is a-receptor sympathomimetic drug exhibiting vasoconstrictive activity and is frequently used in ophthalmology as a mydriatic agent and nasal decongestant in topical formulations. Local complications like conjunctival irritation, corneal edema, or release of iris pigment into the anterior chamber and systemic cardiovascular symptoms such as hypertension and adverse reactions to tropicamide are rare, although allergic contact dermatitis to phenylephrine has been reported on many occasions from different parts of the world. In the largest documented series Herbst et al. performed retrospective analysis of 1641 patients with periorbital dermatitis. Of these, 1053 were diagnosed as allergic periorbital dermatitis and 43 (4.1\%) showed positive patch test reaction to phenylephrine. Borch et al. observed a higher frequency (15\%) of positive reaction to 
phenylephrine in their series of 32 patients. ${ }^{2}$ In contact dermatitis, specifically sensitized CD4 T helper cells provide the immunologic memory that accounts for the more rapid and intense response that occurs after re-exposure with the allergen. ${ }^{4}$

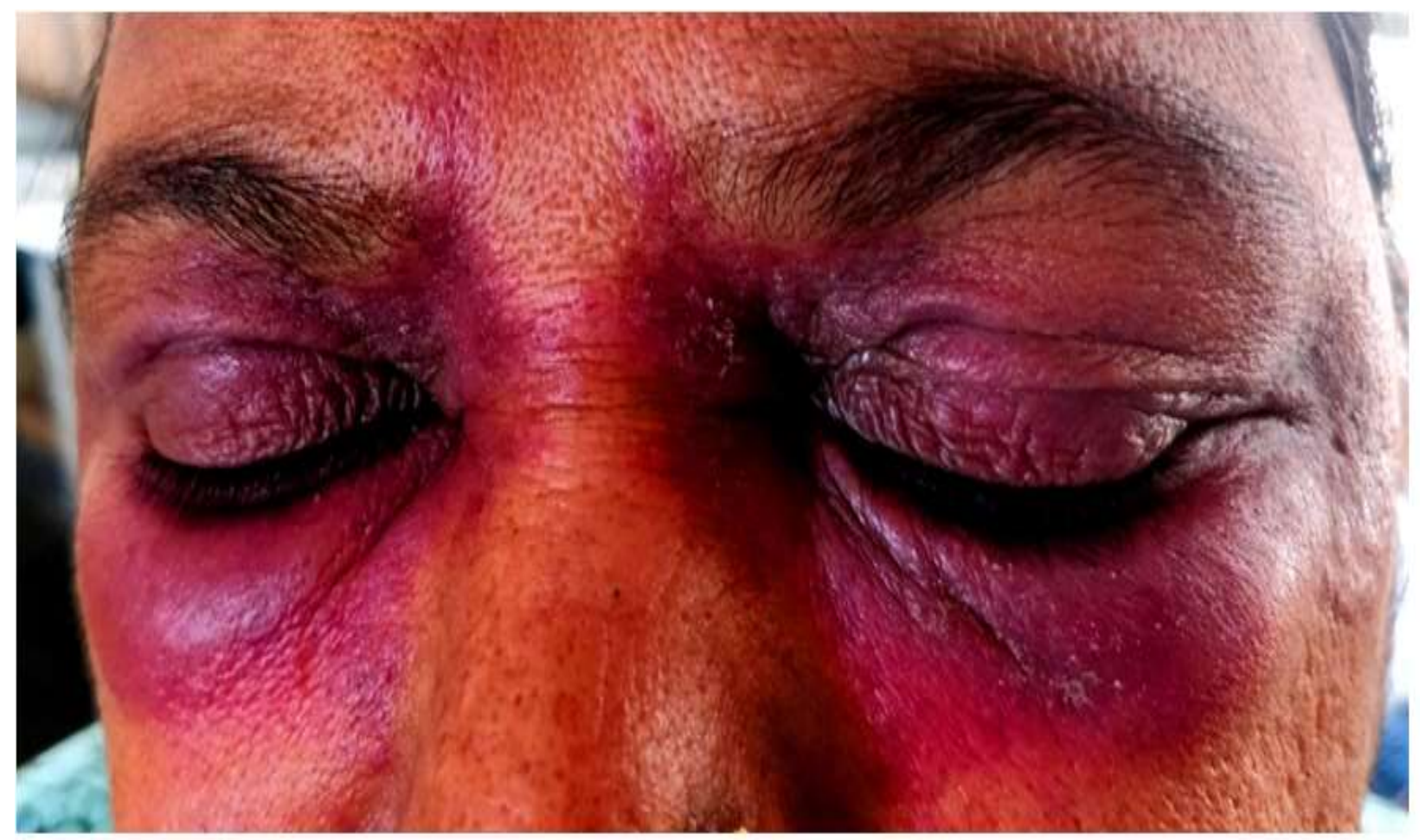

Fig. 01. Erythema and edema of eyelids with conjunctiva congestion.

\section{Conclusion:}

Although uncommon, but the cases of allergic reaction to phenylephrine can occur in some patients. The incidence of allergy caused by the administration of tropicamide $0.8 \% \mathrm{w} / \mathrm{v}+$ phenylephrine $5 \% \mathrm{w} / \mathrm{v}$ eye drop is the case study of a patient presented with a severe contact dermatitis. It is important for all eye care clinicians to realize that adverse effects to diagnostic eye drops are possible and can occur following the most routine of visits. Such reactions can be caused by dilating agents, anesthetics, or preservatives, and these may be allergic or toxic. Clinicians/ophthalmologists should take special care to identify the instigating agent, and if possible to avoid occurrence of such adverse reactions, suggested prior examinations should be performed and use such agents on patients during future exams. Clinicians/Ophthalmologists also should understand how best to manage iatrogenic adverse effects when they encounter them in order to restore a patient's visual function as quickly as possible. 


\section{References:}

1. Bonnie Vu, OD; Arthur Wong, OD; and Susannah Marcus-Freeman, OD. Allergic Reaction to Phenylephrine. Federal practitioner; 2017: 41- 44.

2. Singal A, Rohatgi J, Pandhi D. Allergic contact dermatitis to phenylephrine. Indian J Dermatol Venereol Leprol 2008; 74: 298.

3. J. L. Aggarwal and b. Beveridge I beration of iris pigment in the anterior Chamber Brit.. Ophthal. (1 97 I) 55,544

4. Pragati Garg, Rajiv Garg, Luxmi Singh, Ruby Malhotra, Rajendra Prasad. Allergy to Phenylephrine Hydrochloride Eyedrops: A Case Report. Indian J Allergy Asthma Immunol 2007; 21 (2): 73-76.

5. https://www.drugs.com 